\title{
UTILIZAÇÃO DE DADOS DO SISTEMA DE DESPACHO PARA ESTIMATIVA DE PRODUTIVIDADE DE TRANSPORTE NO PLANO DE LAVRA DE CURTO PRAZO*
}

\section{Resumo}

\author{
Ana Carla de Melo Moreira Campelo ${ }^{1}$ \\ Tatiane Marin ${ }^{2}$ \\ Maria Lúcia Oliveira ${ }^{3}$ \\ Carlos Willian Ferreira dos Santos ${ }^{4}$ \\ Roberto Quadros Menin ${ }^{5}$ \\ Nonato Hugo Campelo da Silva ${ }^{6}$ \\ Aline Lorraine Silva Lacerda
}

Para elaboração de planos de lavra é necessário que se faça o correto dimensionando da capacidade produtiva dos equipamentos envolvidos nas atividades de perfuração, carregamento e transporte. Esse estudo aborda o dimensionamento da produtividade de transporte para planejamento de curto prazo através da utilização de informações oriundas do banco de dados sistema de despacho e distância média de transporte oriunda da confecção dos planos de curto prazo, tomando como base os avanços de lavra e destinação dos materiais. A fim de chegar em um indicador da produtividade de transporte mais fiel aos resultados que de fato são praticados, foram levados em considerações os dados históricos mais recentes, obtidos por meio de um sistema de despacho e controle dos equipamentos (Dispatch $\AA$ )... O trabalho proposto foi aplicado na mesma operação onde os dados foram obtidos e obteve resultados satisfatórios, possibilitando o acompanhamento mais detalhado da execução do plano de curto prazo e demonstrou-se passível de ser aplicado, também, para o planejamento de longo prazo. Os dados utilizados são provenientes de uma mina em operação (Cu-Au) situado no norte do Brasil.

Palavras-chave: Produtividade de transporte; Sistema de Despacho; Estimativa; Plano mensal.

\section{USE OF DATA FROM THE DISPATCHING SYSTEM FOR TRANSPORT PRODUCTIVITY ESTIMATION IN THE SHORT TERM MINING PLAN}

\section{Abstract}

In order to prepare mining plans, it is necessary to do the correct dimensioning of the production capacity of the required equipment in the execution, loading and transportation activities. This study considers the considers the dimensioning of the transport production for short-term planning through the dispatch system and by using the mean transport distance, from the preparation of the short-term plans based on the material that intents to be mined and its destination. In order to provide a more accurate indicator of the transport production, it was taken into account the most recent historical data obtained through a dispatch and control system (Dispatch $\AA$ ). The proposed work was applied in the same operation where the data was obtained and satisfactory results, enabling the detailed monitoring of the execution of the short-term plan and demonstration of liabilities to be applied, also, for long-term planning. The date used is from a mine in operation (Cu-Au) located in the north of Brazil.

Keywords: Transport productivity; Dispatch System; Estimation; Monthly plan.

Engenheira de Minas e Meio Ambiente, mestranda, engenheira de planejamento pleno, planejamento de curto prazo, Vale, Canaã dos Carajás, Pará, Brasil. 
2 Engenheira de Minas, Doutora, Pós doutoranda, Departamento de Engenharia de Minas e de Petróleo, Escola Politécnica da Universidade de São Paulo, São Paulo, Brasil.

3 Engenheira de Minas, mestre, Universidade Federal de Campina Grande, Campina Grande, Paraíba, Brasil.

4 Engenheiro de Minas e Meio Ambiente, graduando, Universidade Federal do Sul e Sudeste do Pará, Marabá, Pará, Brasil.

5 Engenheiro de Minas, mestre, engenheiro geoestatístico, planejamento de longo prazo, Vale, Canaã dos Carajás, Pará, Brasil.

6 Engenheiro de Minas e Meio Ambiente, bacharel, engenheiro de planejamento sênior, planejamento de curto prazo, Vale, Marabá, Pará, Brasil.

7 Engenheira de Minas, bacharel, engenheira de despacho eletrônico, Vale, Canaã dos Carajás, Pará, Brasil. 


\section{INTRODUÇÃO}

Com os últimos desenvolvimentos em tecnologia, é possível monitorar continuamente as várias atividades dos equipamentos, gravando e armazenando seus indicadores de performance em bancos de dados, visando a centralização de dados e integração para uma análise mais elaborada². $\mathrm{E}$ a indústria de mineração está sempre procurando novos desafios na otimização de seus processos e produtividade de seus equipamentos.

$\mathrm{Na}$ área de planejamento de lavra a pesquisa operacional é utilizada deste os anos $1960{ }^{4}$. Técnicas de simulação para representar a operação, incluindo a utilização de dados de despacho são utilizadas comumente ${ }^{5}$. Entretanto na indústria mineral brasileira o planejamento mensal ainda utiliza indicadores pouco realísticos. Este trabalho propõe uma abordagem prática e simples para trazer dados mais realísticos no planejamento de lavra mensal.

O dimensionamento dos equipamentos, utilizados em um empreendimento mineiro, faz uso de uma série de indicadores referentes a capacidade de carregamento e transporte. A correta estimativa de indicadores como a disponibilidade física, a utilização física e a produtividade, são de fundamental importância para as decisões à serem tomadas. Um cenário de curto prazo demanda uma abordagem diferente dos indicadores em relação à um plano de longo prazo, visto que são estes indicadores que irão definir a capacidade de produção em um espaço temporal menor, aonde os valores médios do longo prazo não podem ser utilizados.

Para que o plano de curto prazo se torne factível é preciso que se realize adaptações nesses indicadores, em comparação aos utilizados no plano de longo prazo. De maneira geral, se faz necessário inserir no plano de curto prazo informações que representem o cenário presente da operação. Ao considerarmos a disponibilidade física, se leva em consideração o tempo que os equipamentos estarão disponíveis. Essa informação é obtida considerando as inspeções mais recentes dos equipamentos. Da mesma forma o plano de revitalização destes equipamentos também é considerado.

Tratando-se do dimensionamento dos indicadores de utilização física e produtividade, principalmente das frotas de transporte, é pratica comum a utilização de dados históricos mais recentes ao plano de lavra em questão, por exemplo, no plano mensal normalmente é utilizado o valor do mês que antecedeu ao mês que se quer planejar.

Entretanto, ao utilizar dados de produtividade médios, não se leva em consideração a influência da distância média de transporte (DMT) no resultado executado. A DMT é calculada entre os pontos de carregamento e basculamento de material, e pode variar de acordo com as necessidades operacionais do projeto.

Esse trabalho tem por objetivo fazer uma análise descritiva sobre todos os componentes que podem ser extraídos do banco de dados do despacho e plano de lavra de curto prazo para estimativa da produtividade de transporte do plano mensal subsequente. Como base para este estudo foram utilizados dados reais de produção de uma mina de cobre no norte do Brasil, a qual utiliza como software de otimização de equipamentos Dispatch ${ }^{\circledR}$ fornecido pela Modular Mining System do Brasil. 


\section{MATERIAIS E MÉTODOS}

A metodologia utilizada nesse estudo de caso é composta por quatro etapas: coleta e tratamento de dados do sistema de despacho, confecção do plano de lavra mensal, estimativa da produtividade e verificação dos resultados.

\subsection{Coleta e Tratamento de Dados}

Com o foco em um dimensionamento de curto prazo, foram coletados dados históricos do banco de dados (BD) do sistema de despacho de um período de até quatro meses. Entretanto foi dado maior peso nos dados do mês imediatamente anterior ao momento da execução do plano mensal. Estes dados são:

Tabela 1. Dados extraídos do BD do despacho

\begin{tabular}{lc}
\hline INDICADOR & SIGLA \\
\hline Tempo de Viagem Vazio & TVV \\
\hline Tempo de Fila para Carregar & TFC \\
\hline Tempo de Manobra para Carregar & TMC \\
\hline Tempo de Carregamento & TC \\
\hline Tempo de Viagem Cheio & TVC \\
\hline Tempo de Fila para Bascular & TFB \\
\hline Tempo de Manobra para Bascular & TMB \\
\hline Tempo de Basculamento & TB \\
\hline Carga Média & CM \\
\hline Local de Carregamento & LOCC \\
\hline Local de Basculamento & LOCB \\
\hline Hora Trabalhada & HT \\
\hline Distância Média de Transporte & DMT \\
\hline
\end{tabular}

Para tratamento dos dados do tempo de ciclo de transporte, foi utilizado o software Excel, fragmentados em sua menor unidade disponível, conforme Tabela 1, através de média simples. Nesse trabalho não foram retirados os valores anômalos ou zerados.

Todos os tempos do ciclo de transporte foram estratificados por frota de equipamento de carga e frotas de transporte. Essa estratificação se faz necessária para análise dos dados, visto que as diferentes frotas de equipamento possuem diferentes capacidades e analisa-las em conjunto poderia inferir em resultados inadequados.

Os tempos do ciclo de transporte se dividem em tempos fixos e variáveis ${ }^{1-3}$, conforme Figura 2. 


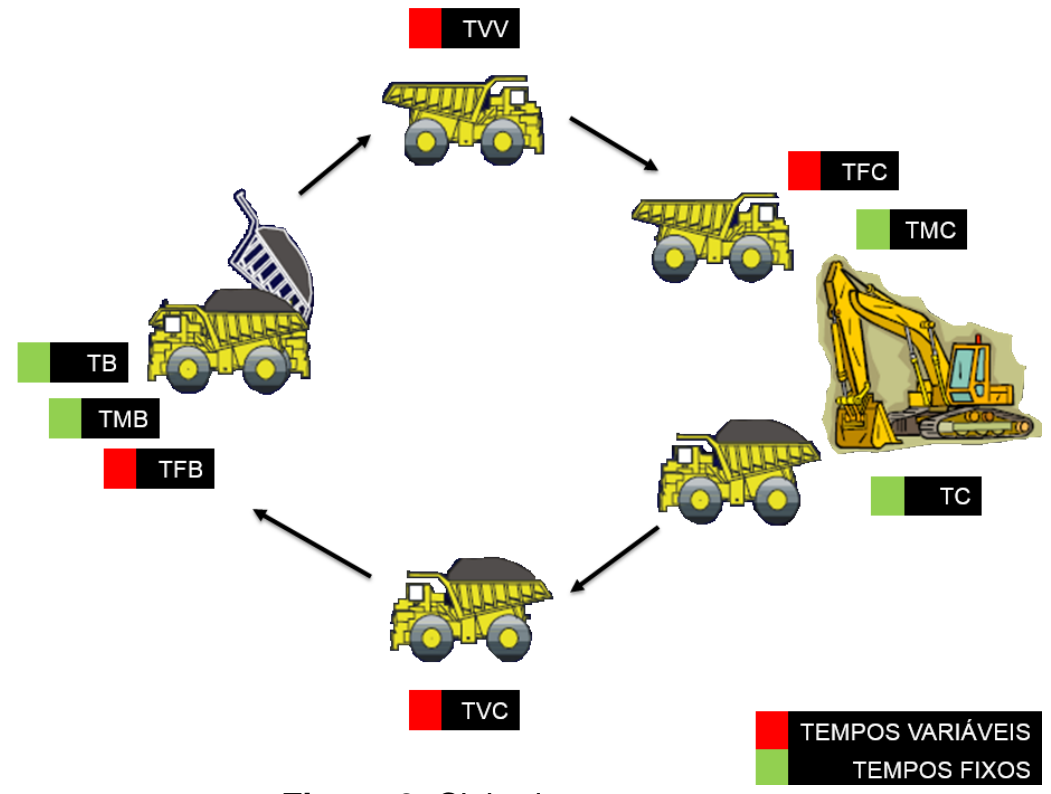

Figura 2. Ciclo de transporte.

Os tempos fixos possuem essa denominação porque, teoricamente, tem que possuir um número constante se tratarmos de uma escavadeira e um caminhão específico, por exemplo uma escavadeira PH4100 para um caminhão CAT793, porém de uma forma geral existem outras interferências que podem fazer com que esse tempo tenha uma pequena oscilação como por exemplo: habilidade do operador da escavadeira, fragmentação do material a ser carregado e o desempenho do equipamento de carregamento.

Parar calcular a produtividade de transporte variando a DMT é necessário que se utilize o tempo da hora trabalhada do equipamento de transporte na menor unidade possível pois produtividade é dada pela Equação 1:

Produtividade de transporte $=\frac{\text { produção }}{H T}$

Para extração do indicador de desempenho (KPI) produtividade de transporte se utilizam duas consultas SQL no servidor do despacho conforme diagramas das Figuras 2 e 3 . Dessa forma podem ser extraídos os dados de produção e HT, respectivamente. Quando se utiliza a consulta no formato do esquema da Figura 3 é trazido como resultado a HT total, pois é utilizado a classificação do estado do equipamento que pode ser divido em: hora de manutenção, hora trabalhada e hora improdutiva. 


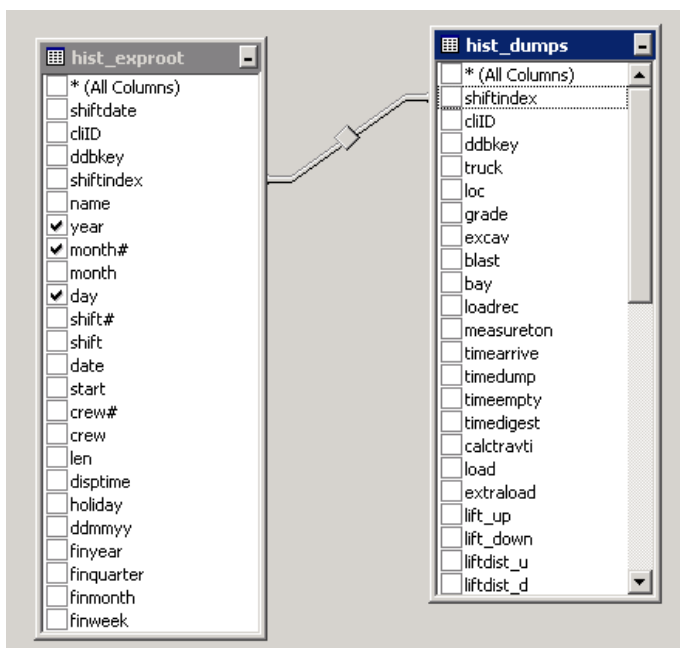

Figura 2. Diagrama representativo da consulta SQL de produção de transporte

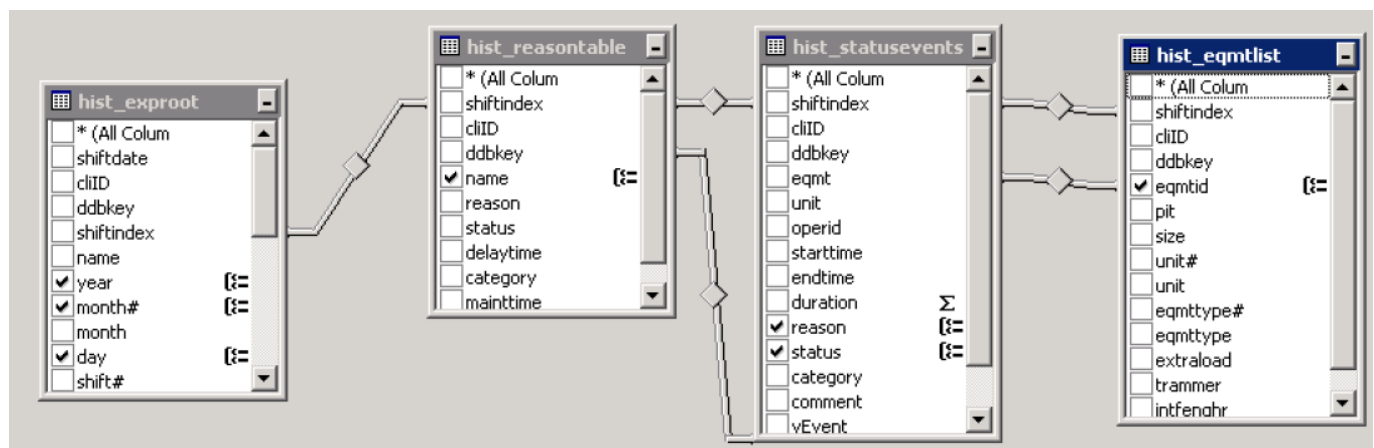

Figura 3. Diagrama representativo da consulta SQL HT da tabela de estados

Entretanto quando o que se deseja é fazer uma variação da DMT para gerar variação na $\mathrm{HT}$, então é preciso trabalhar a $\mathrm{HT}$ em todos os seus componentes conforme Equação 2:

$H T=T V V+T F C+T M C+T C+T V C+T F B+T M B+T B+((H T) *$ fator de correção))

Esses dados são extraídos do BD do sistema de despacho através de um diagrama de consulta SQL conforme a Figura 4. Dessa forma a HT passa a ser constituída por todos os tempos discriminados que compõem o ciclo de transporte, todos são retirados das tabelas de carga e transporte. Mas, por uma característica do sistema de despacho que é utilizado na mina do estudo de caso, quando comparadas essas duas forma de extração da HT elas não ficam iguais, por esse motivo se faz necessário o acréscimo de um fator de correção que é obtido através da diferença entres essas duas formas de adquirir a HT, conforme Equação 3. Em nosso estudo o fator de correção gira em torno de $6 \%$.

Fator de correção $=\left(H T^{1}-H T^{2}\right) / H T^{1}$

Onde,

$\mathrm{HT}^{1}=$ Hora trabalhada da tabela de estados 
$\mathrm{HT}^{2}=$ Hora trabalhada originada das tabelas de carga e transporte constituída pelos tempos discriminados que compõem o tempo de ciclo

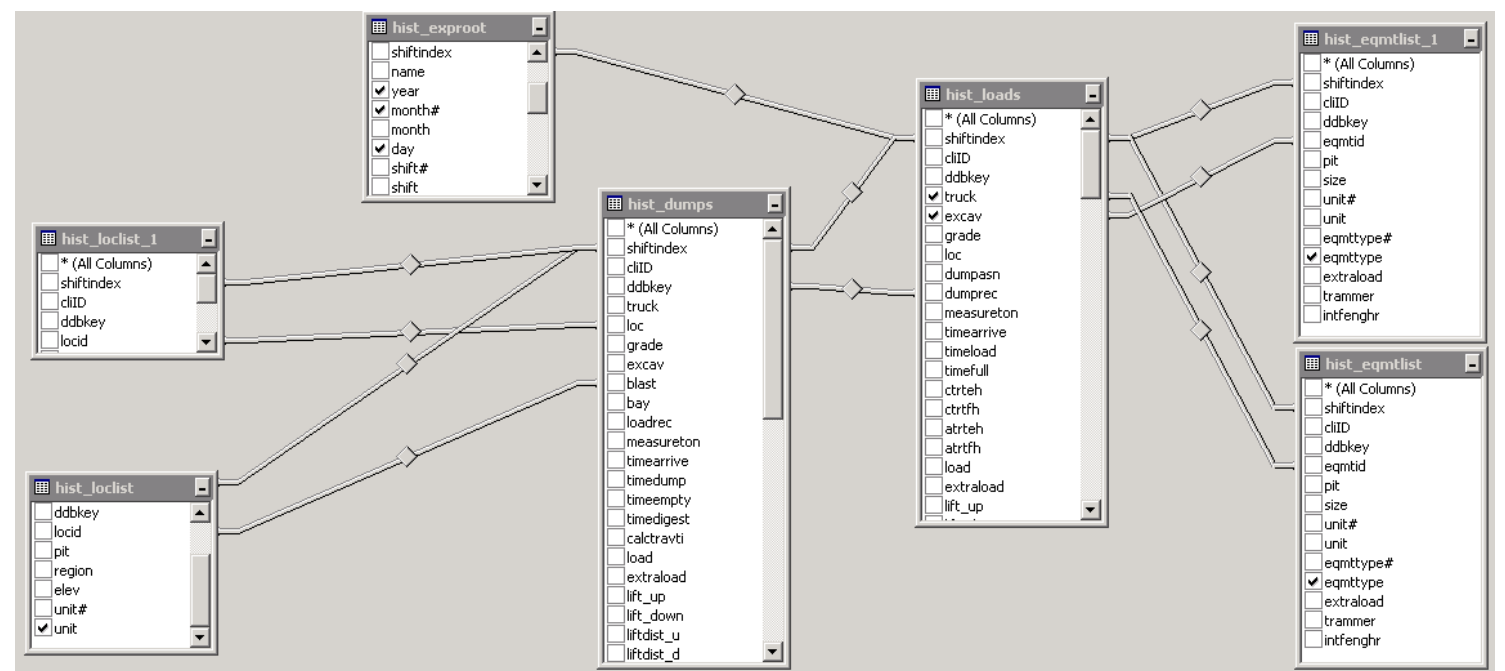

Figura 4. Diagrama representativo da consulta SQL HT da tabela de carga e transporte

\subsection{Confecção do plano de lavra mensal}

Durante a confecção do plano são definidos os avanços de lavra para cada equipamento de carga, bem como os avanços nos pontos de basculamento (depósitos de estéril e pilhas de minério). De posse dessas duas informações se pode medir a DMT esperada para o mês entre todos os pontos de carregamento e basculamento. Essa medida é feita no software de planejamento que se utiliza, no exemplo da Figura 1 foi feito no software Gemcom.

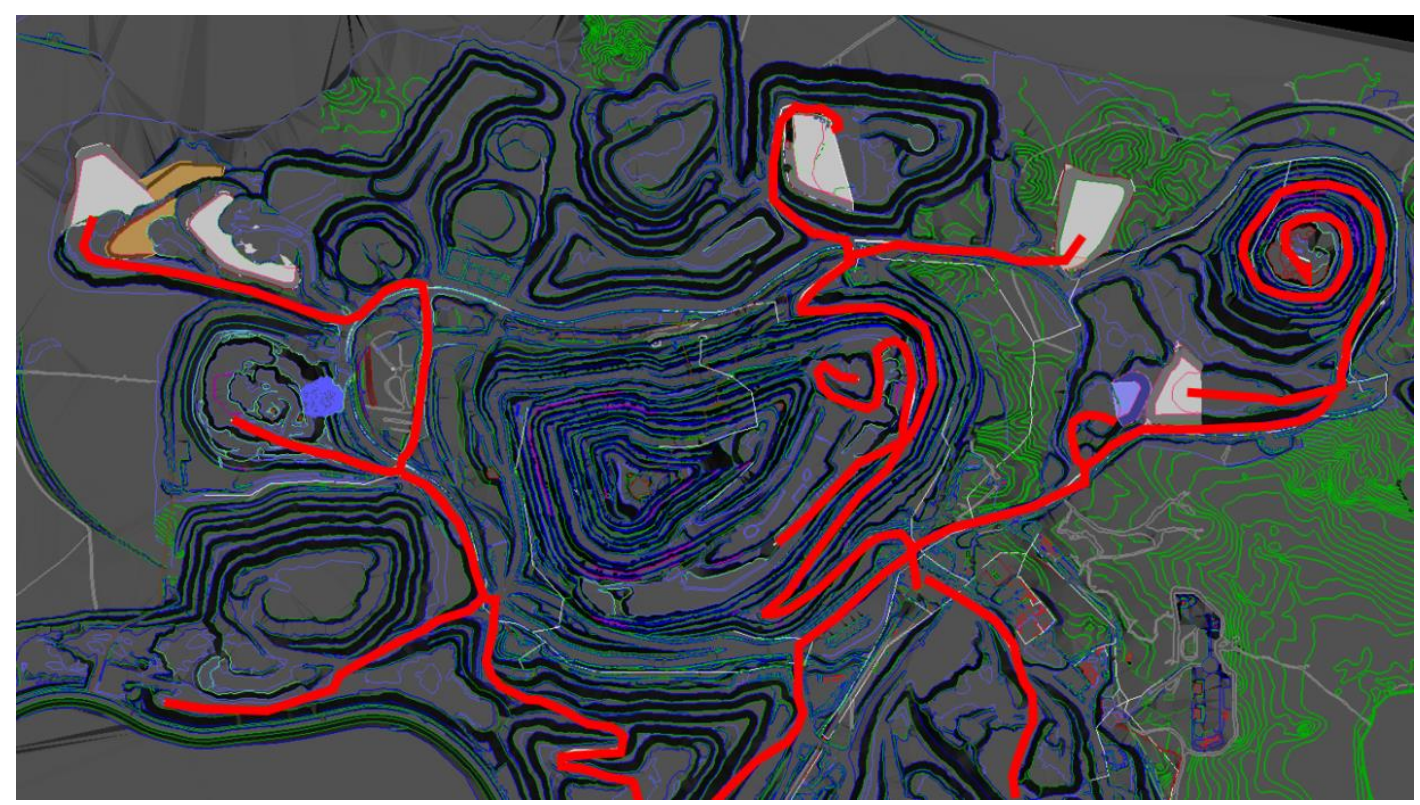

Figura 1. Caminhamento pelos acessos provenientes do plano mensal

\subsection{Estimativa da produtividade}

Os tempos de viagem (cheio/vazio) e DMT podem ser extraídos diretamente do sistema de despacho. E com isso é possível obter a velocidade realizada. Para 
efeito da simulação do mês subsequente não se pode utilizar a DMT histórica, a menos que seja para comparação. Sendo assim, de posse da velocidade histórica mais próxima (mês corrente) e DMT medida no software de planejamento (mês subsequente) pode-se obter o tempo de transporte estimado para o próximo mês e com ele chegar no objetivo final que é a produtividade para o mês subsequente.

Vale ressaltar que dentre todas as unidades de tempo que constituem a HT, os tempos de viagem (TVV e TVC) recebem um destaque especial, pois o mesmos, nesse estudo de caso, são responsáveis por $65 \%$ do tempo total do ciclo.

Considerados toda informação exposta até aqui, o tempo de transporte para esse estudo é dado pela Equação 4. E a produtividade de transporte é dado pela Equação 5.

$\left(\frac{D M T}{\text { velocidade média }}\right)=T V C+T V V$

Produtividade de transporte $=\frac{\text { carga média }}{\left(T M C+T M B+T F C+T F B+T C+T B+\left(\frac{D M T}{\text { velocidade média }}\right)\right)}$

Para alcançar o detalhamento necessário no dimensionamento de produtividade de transporte para o plano de lavra mensal, esses dados foram estratificados por equipamento de carga, frota de transporte, local de carregamento e local de basculamento.

\subsection{Verificação dos resultados}

Após a conclusão do dimensionamento do mês subsequente foi feito um acompanhamento dos dados realizados para verificação da possibilidade real de utilização dessa metodologia para dimensionamento de produtividade, verificação de possíveis lacunas e oportunidades de melhoria.

\section{RESULTADOS E DISCUSSÃO}

O primeiro resultado obtido foi a estimativa da produtividade de transporte para o mês subsequente, conforme Tabela 2.

Tabela 2. Estimativa da produtividade de transporte

\begin{tabular}{|c|c|c|c|c|c|c|c|}
\hline & MASSA (TON) & $\begin{array}{l}\text { ADOS REALIZAL } \\
\text { DMT (KM) }\end{array}$ & $\begin{array}{c}\text { S NO MÊS CORRENTE } \\
\text { PRODUTIVIDADE } \\
\text { TON/H }\end{array}$ & $\begin{array}{l}\text { VELOCIDADE } \\
\text { (KM/H) }\end{array}$ & $\begin{array}{l}\text { MASSA TRANSPORTE } \\
\text { MÊS SUBSEQUENTE } \\
\text { (TON) }\end{array}$ & $\begin{array}{c}\text { DMT MÊS } \\
\text { SUBSEQUENTE (KM) }\end{array}$ & $\begin{array}{l}\text { PRODUTIVIDADE MÊS } \\
\text { SUBSEQUENTE (TON/H) }\end{array}$ \\
\hline ESC1 & $43,205.04$ & 2.56 & 356 & 18.84 & $42,184.54$ & 2.83 & 330 \\
\hline ESC2 & - & & & & - & - & - \\
\hline ESC3 & $91,102.75$ & 3.30 & 265 & 16.87 & $84,654.17$ & 2.93 & 291 \\
\hline ESC4 & $459,714.33$ & 2.62 & 232 & 17.10 & $297,874.77$ & 4.06 & 173 \\
\hline FROTA DE TRANSPORTE A ESC5 & $147,257.08$ & 3.95 & 212 & 17.33 & $175,044.52$ & 4.06 & 207 \\
\hline ESC6 & $98,443.21$ & 3.02 & 226 & 15.49 & $96,175.07$ & 1.60 & 338 \\
\hline ESC7 & $56,365.17$ & 2.29 & 282 & 16.08 & $116,844.79$ & 3.03 & 234 \\
\hline ESC8 & $238,222.38$ & 1.58 & 386 & 17.11 & $191,767.18$ & 2.81 & 269 \\
\hline & $1,229,189.01$ & 2.55 & 262 & 16.97 & $1,098,145.04$ & 3.12 & 245 \\
\hline ESC1 & $1,119,418.25$ & 2.55 & 576 & 20.04 & $1,092,977.54$ & 2.83 & 532 \\
\hline ESC2 & - & & & & $660,142.08$ & 2.53 & 474 \\
\hline ESC3 & $1,263,152.17$ & 3.35 & 421 & 17.67 & $1,173,741.67$ & 2.93 & 466 \\
\hline ESC4 & $120,816.90$ & 3.61 & 283 & 18.15 & $78,284.06$ & 4.06 & 263 \\
\hline FROTA DE TRANSPORTE B ESC5 & $345,979.49$ & 4.13 & 329 & 18.28 & $411,265.88$ & 4.06 & 332 \\
\hline ESC6 & $398,126.74$ & 3.13 & 348 & 17.00 & $388,953.85$ & 1.60 & 501 \\
\hline ESC7 & $109,342.79$ & 2.76 & 382 & 17.75 & $226,667.21$ & 2.75 & 382 \\
\hline ESC8 & $188,504.69$ & 2.62 & 376 & 17.96 & $151,744.82$ & 2.62 & 376 \\
\hline & $3,545,341.03$ & 3.10 & 425 & 18.27 & $4,183,777.12$ & 2.83 & 463 \\
\hline
\end{tabular}


$\mathrm{Na}$ Tabela 2 é exibido a produtividade estimada para as frotas de transporte $\mathrm{A}$ e $\mathrm{B}$, sendo de 245 toneladas/hora e 463 toneladas/hora, respectivamente. Para a frota $A$ o mês subsequente teria uma produtividade de transporte estimada menor do que 0 mês anterior, e recebe clara influência da DMT, a qual no mês anterior foi de 2.55 $\mathrm{km}$ e no mês subsequente estima-se $3.12 \mathrm{~km}$. Para a frota $B$ haveria um aumento de produtividade (425 ton/h $\rightarrow 463$ ton $/ \mathrm{h}$ ) no mês subsequente em função da redução de DMT $(3.10 \mathrm{~km} \rightarrow 2.83 \mathrm{~km})$.

As velocidades de transporte utilizadas, carga média por viagem, tempos de fila e todos os tempos fixos foram os que efetivamente ocorreram no mês anterior ao planejamento, ou seja, aproxima muito da realidade, é como se "considerasse" o perfil de transporte.

Na Tabela 3, é exibido o detalhe da estimativa de produtividade de transporte da frota de transporte $B$ carregado na escavadeira $A$, conforme todos os locais de basculamento que foram destinados para o avanço dessa escavadeira.

Tabela 3. Estimativa da produtividade de transporte por local de basculamento

\begin{tabular}{|c|c|c|c|c|c|c|c|c|c|c|c|}
\hline A & B & C & D & E & $\mathrm{F}$ & G & $\mathrm{H}$ & I & J & $\mathrm{K}$ & L \\
\hline & & LOCAL DE BASCULO & MASSA (TON) & $\begin{array}{r}\text { DAD } \\
\text { DMT (KM) }\end{array}$ & $\begin{array}{l}\text { OS REALIZADOS NO MÊs C } \\
\text { \% DA MASSA PARA ESSE } \\
\text { LOCAL DE BASCUIO }\end{array}$ & $\begin{array}{l}\text { ORRENTE } \\
\text { PRODUTIVIDADE } \\
\text { (TON/H) }\end{array}$ & $\begin{array}{l}\text { VELIOCIDADE } \\
\text { (KM/H) }\end{array}$ & $\begin{array}{l}\text { \% DA MASSA PARA ESSE } \\
\text { LOCAL DE BASCULO }\end{array}$ & $\begin{array}{l}\text { MASSA TRANSPORTE } \\
\text { MÊS SUBSEQUENTE } \\
\text { (TON) }\end{array}$ & $\begin{array}{l}\text { DMT MÊS SUBSEQUENTE } \\
\text { (KM) }\end{array}$ & $\begin{array}{l}\text { PRODUTIVIDADE MÊS } \\
\text { SUBSEQUENTE (TON/H) }\end{array}$ \\
\hline \multirow{23}{*}{ 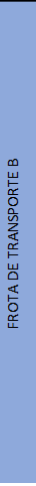 } & \multirow{23}{*}{ ESC1 } & ACESSO_OFIC_CENTRH & 432.70 & 4.16 & $0 \%$ & 557 & 29.19 & & & & - \\
\hline & & BRIT_METSO_250 & $\begin{array}{r}425.42 \\
608604\end{array}$ & 3.52 & $0 \%$ & 458 & 21.13 & & & & \\
\hline & & $\begin{array}{l}\text { BRIIADOR } \\
\text { NOBDESTE3 } 280\end{array}$ & $6,286.04$ & 3.18 & $1 \%$ & 520 & 22.99 & & & & - \\
\hline & & NORDESTES 3 N1 260 & $\begin{array}{r}7,443.24 \\
21807.18\end{array}$ & 3.51 & $1 \%$ & 518 & 21.25 & & & & - \\
\hline & & NORTE-245 & $67,698.84$ & 2.65 & $6 \%$ & 593 & 21.38 & $37 \%$ & $400,758.43$ & 298 & 544 \\
\hline & & NORTE-255 & $\begin{array}{r}\quad 142,264.32 \\
\end{array}$ & 2.27 & $13 \%$ & 665 & 20.66 & $15 \%$ & $163,946.63$ & 2.75 & 577 \\
\hline & & NORTE-260 & 210.80 & 2.18 & $0 \%$ & 514 & 23.30 & & & & - \\
\hline & & NORTE-265 & $243,791.70$ & 2.52 & $22 \%$ & 558 & 19.52 & $19 \%$ & $209,487.36$ & 2.55 & 552 \\
\hline & & REM_PIS1-184 & $4,958.72$ & 1.35 & $0 \%$ & 747 & 22.76 & & & & - \\
\hline & & REM_PIS1-200 & 599.00 & 2.30 & $0 \%$ & 706 & 20.70 & & & & \\
\hline & & REM PIS1-2160 & $1,173.70$ & 1.19 & $0 \%$ & 658 & 9.22 & & & & . \\
\hline & & SUDESTE2-275 & 179.40 & 2.05 & $0 \%$ & 561 & 16.16 & & & & - \\
\hline & & SUDOESTE-265-BARRA & $12,982.54$ & 2.32 & $1 \%$ & 599 & 18.80 & & & & - \\
\hline & & SUDOESTE-275 & $500,541.42$ & 2.40 & $45 \%$ & 582 & 18.89 & & & & - \\
\hline & & SULFETADO AT/B & $31,686.74$ & 3.50 & $3 \%$ & 529 & 23.30 & & & & - \\
\hline & & SULFETADO SAT4 & $1,083.80$ & 3.43 & $0 \%$ & 499 & 22.08 & & & & - \\
\hline & & SULFETADO SATP & $12,603.94$ & 3.91 & $1 \%$ & 470 & 23.87 & & & & \\
\hline & & SULFETADO SBT/C & $3,216.15$ & 3.80 & $0 \%$ & 425 & 22.33 & & & & - \\
\hline & & SW-245_FLAUTA & $46,349.84$ & 3.86 & $4 \%$ & 423 & 25.06 & & & & \\
\hline & & GRELHA & & & $0 \%$ & & 18.89 & $13 \%$ & $136,622.19$ & 2.69 & 531 \\
\hline & & SW 325 & - & & $0 \%$ & & 18.80 & $17 \%$ & $182,162.92$ & 2.99 & 487 \\
\hline & & & $13,682.76$ & 1.20 & $1 \%$ & 1,364 & 26.27 & & & & \\
\hline & & & 1,119,418.25 & 2.55 & $100 \%$ & 576 & 20.04 & $100 \%$ & $1,092,977.54$ & 2.83 & 532 \\
\hline
\end{tabular}

Na coluna C (local de básculo) com preenchimento de amarelo são exibido todos os locais onde ocorreu basculamento no mês anterior, e na coluna $F$ são exibidos os percentuais da massa em cada um desses locais. Os locais de basculamento que estão com bordas são os locais que foram definidos no plano para basculamento no mês subsequente, e seus respectivos percentuais de massa estão na coluna I. Teremos a DMT estimada para cada um dos pontos de basculamento definidos para o mês subsequente na coluna $\mathrm{K}$, assim como as produtividades nas coluna $\mathrm{L}$.

Para os locais de basculamento que não houve deposição no mês anterior, é necessário que seja escolhido a velocidade média para que os cálculos sejam executados, dessa forma foram escolhidos aqueles que são mais próximos desse ponto de basculamento ou que possuem perfil de transporte semelhante.

Um segundo resultado obtido por esse trabalho é a possibilidade de acompanhamento detalhado do plano de deposição (DMT e perfil de transporte) e seu impacto sobre a produtividade de transporte.

Nos Gráficos 1 e 2, são apresentados o acompanhamento da DMT e velocidade média, programados e realizados para ESC1 frota de transporte B. Do dia 07 ao dia 
19 esse equipamento esteve em manutenção preventiva, por isso não são apresentados valores.

Gráfico 1. DMT X Produtividade de transporte ESC1 frota de transporte $B$

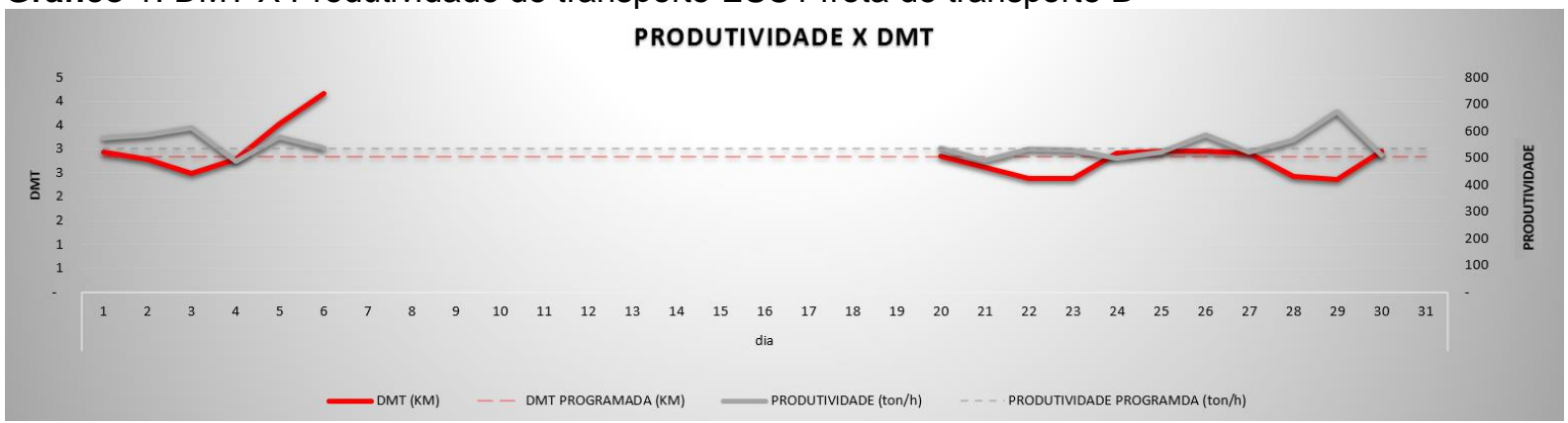

Gráfico 2. Velocidade média X Produtividade de transporte ESC1 frota de transporte $B$

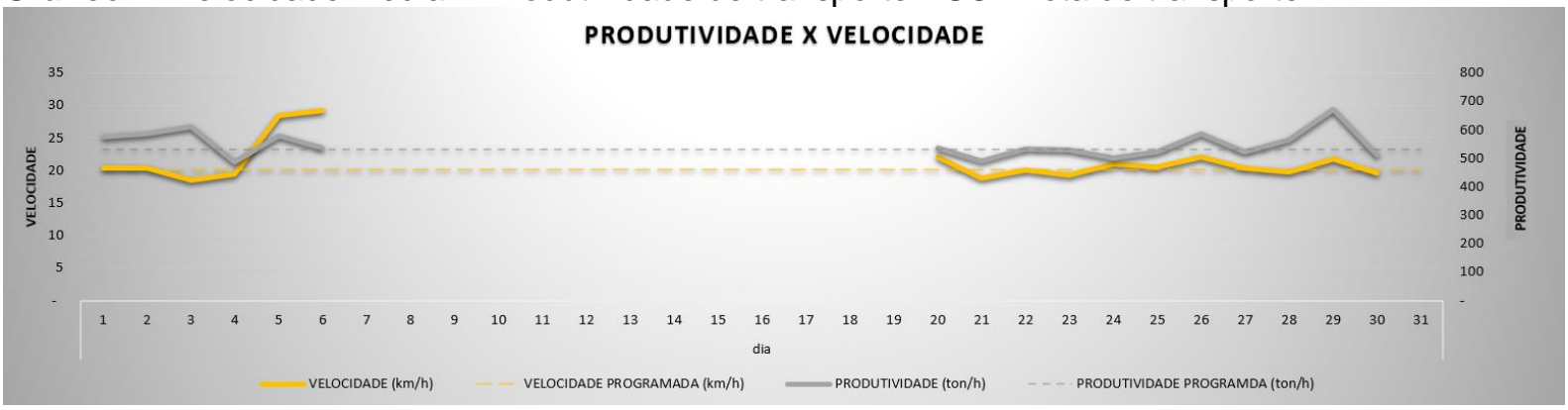

No Gráfico 3 podemos observar, ainda para ESC1, o quão aderente foi o realizado com relação ao planejado. A DMT realizada foi de $2.84 \mathrm{~km}$ para um planejado de $2.83 \mathrm{~km}$. Pode-se fazer o acompanhamento detalhado de todos os desvios pois, conforme pode ser visto no Gráfico 3, é possível verificar que houve deposição em local que não havia sido programado e houve também diferença nos percentuais destinados, por exemplo, no depósito N-265 que havia sido programado apenas $19 \%$ do material acabou sendo executado $33 \%$, e certamente isso exerce influência na DMT.

Gráfico 3. Percentual de massa destinado para locais de deposição - planejado x realizado 


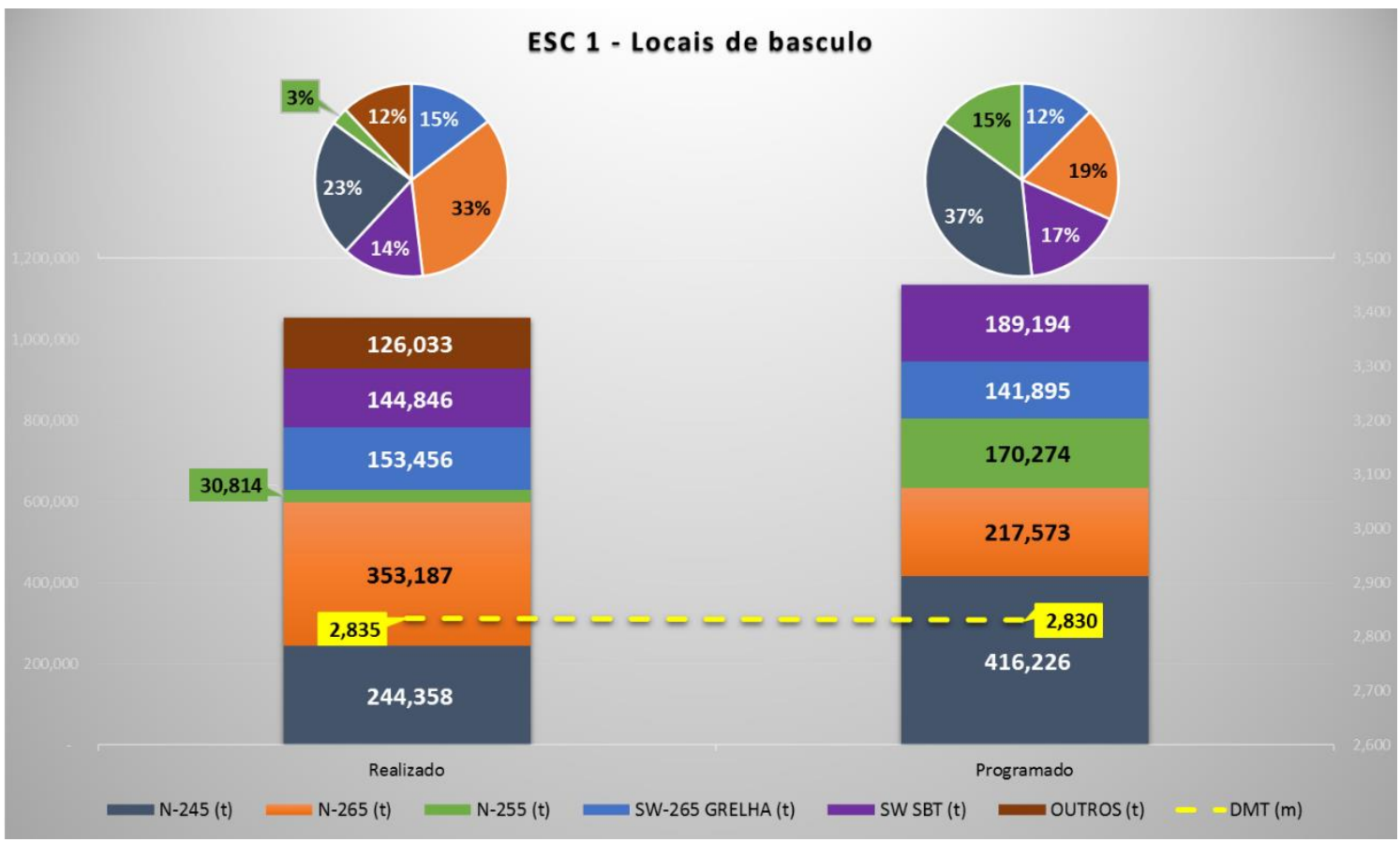

A mesma análise foi feita para cada um do equipamentos de cargas e frota de transporte.

\section{CONCLUSÃo}

Foi constatado a eficácia do dimensionamento de produtividade de transporte através da utilização de dados históricos do BD do despacho e estimativa de DMT oriunda do plano de lavra.

Apesar da estimativa ser realista o resultado da produtividade do mês subsequente não é exatamente o programado, principalmente em função desvios ligados às condições operacionais que não foram previstas no plano. No entanto, se aproximar muito do planejado, conforme pudemos verificar no caso da ESC1.

A utilização dos dados históricos dos tempos fixos que constituem de ciclo do mês anterior dá uma segurança maior no plano visto que são os dados reais.

Além de um dimensionamento de produtividade mais acurado, surge a possibilidade de acompanhamento detalhado dos desvios, como deposição em locais que não haviam sido programados ou diferença dos percentuais destinados, como pôde ser observado no Gráfico 3. Essa avaliação recebe um destaque especial pois se o dimensionamento de produtividade de transporte fosse feito como antes, apenas como a média da produtividade executada do mês antecessor, não se teria detalhe nenhum para acompanhamento dos desvios e verificação de oportunidades de melhoria.

Sugere-se que essa pesquisa seja estendida para dimensionamento de produtividade no planejamento de longo prazo, o qual normalmente se utiliza de dados de tempos fixos do tempo de ciclo de transporte baseados nos manuais dos fornecedores, se sugere que há oportunidades de melhoria na acurácia do 
dimensionamento buscando os dados históricos e fazendo análise estatística mais detalhada dos mesmos.

\section{REFERÊNCIAS}

1 CATERPILLAR. Caterpillar performance handbook. Edition 43. Caterpillar. Illinois, 2013.

2 CORONADO, P. P. V., TENORIO, V. O. Optimization of open pit haulage cycle using a KPI controlling alert system and a discrete-event operations simulator. Mining and Geological Engineering Department/University of Arizona. Arizona, 2015.

3 DARLING, P. SME MINING ENGINEERING HANDBOOK. 3rd Edition. Society for Mining, Metallurgy, and Exploration. Colorado, 2011.

4 NEWMAN, Alexandra M. et al. A review of operations research in mine planning. Interfaces, v. 40, n. 3, p. 222-245, 2010.

5 TAN, Yifei et al. Operations modeling and analysis of open pit copper mining using GPS tracking data. In: Proceedings of the Winter Simulation Conference. Winter Simulation Conference, 2012. p. 117. 